\title{
Traumatic Unilateral Sixth to Seventh Cervical Vertebral Facet Luxation in a Dog
}

\author{
Philemon Karli ${ }^{1}$ Martin Bass ${ }^{2}$ Roman Inauen ${ }^{2}$ Danielle Bass ${ }^{3}$ \\ 1 Department of Neurology, VET Zentrum AG, Pfungen, Zürich, Switzerland \\ ${ }^{2}$ Department of Surgery, VET Zentrum AG, Pfungen, Zürich, Switzerland \\ 3 Department of Diagnostic Imaging, VET Zentrum AG, Pfungen, \\ Zürich, Switzerland \\ Address for correspondence Philemon Karli, Department of \\ Neurology, VET Zentrum AG, Riedäckerstrasse 7, 8422, Pfungen, \\ Zürich, Switzerland (e-mail: pkarli@vetzentrum.ch).
}

VCOT Open 2018;1:e19-e24.

\begin{abstract}
Keywords

- computed tomography

- open reduction

- canine

- cervical spine

- trauma

We describe the surgical reduction of a traumatic, unilateral cervical vertebral facet joint luxation in a large breed dog. Computed tomography (CT) including CT myelography revealed complete luxation of the left articular processes $\mathrm{C} 6$ to $\mathrm{C} 7$ without fracture of the main weight-bearing structures. There was marked spinal cord compression secondary to the malalignment. Due to the non-ambulatory tetraparetic status, open reduction was chosen as the treatment of choice. After making a dorsal surgical approach to the caudal cervical spine, the luxated articular processes were visible and repositioned. Owing to marked mobility of this joint, temporary stabilization with a nonabsorbable suture between the spinous processes of $\mathrm{C} 6$ and $\mathrm{C} 7$ was performed and good stability was obtained. The dog recovered uneventfully from the surgery and regained normal ambulation. Follow-up examination including a second CT 9 months after surgery showed no neurological abnormalities and unrestricted motion of the neck. Normal cervical vertebral alignment with moderate osteoarthritis of the right facet joint and mild spondylosis deformans C6 to C7 were present. The present case report shows successful management of a traumatic cervical facet joint luxation without persistent neurological deficits. Open reduction and temporary stabilization with suture material seem to be a valuable method of treatment. The dorsal approach allowed direct inspection of the facet joints with concurrent access to place suture material for temporary stabilization.
\end{abstract}

\section{Introduction}

Vertebral fractures and luxations account for $7.1 \%$ of dogs diagnosed with neurological problems. ${ }^{1}$ The thoracolumbar ${ }^{2,3}$ and the lumbar ${ }^{4}$ vertebral column are reported to be most commonly affected in canine patients. The cervical area is less prone to fracture or luxation, and is affected in 7 to $20 \%$ of the cases. ${ }^{2,4,5}$ The caudal cervical spine was more commonly affected in one study ${ }^{2}$ and larger $(>15 \mathrm{~kg})$ dogs were reported to be over-represented in a further study. ${ }^{6}$ Pure luxations of vertebrae have a relatively low incidence with $20 \%$ in one study $^{2}$ and even less in small dogs and cats. Larger dogs, therefore, have a higher risk for vertebral luxation, most likely because the bone is relatively thicker and less prone to

received

September 19, 2018

accepted

September 19, 2018
DOI https://doi.org/

10.1055/s-0038-1676323. ISSN 2625-2325. fractures. Torsional forces are the common cause of luxations, although frequently they are accompanied by fractures of those structures. ${ }^{7}$ We report a complete unilateral luxation of the left sixth to seventh cervical vertebral (C6-C7) facet joint, with dorsal displacement of the cranial articular process and subsequent open surgical reduction without partial facetectomy and temporary dorsal stabilization in a dog.

\section{History and Clinical Presentation}

A 7-year-old female Border Collie was presented because of trauma-related tetraparesis. The dog presumably got stuck in an electric sheep net while chasing an animal. Occurring in darkness, the event was not observed, but the owner was
License terms

Stuttgart · New York

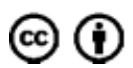


alerted by the dog screaming. Due to subsequent inability to walk, the dog was presented to the veterinarian. The dog was initially treated with intravenous fluids and analgesics. Due to persistent tetraparesis and generalized increased extensor tone, a cervical spinal lesion was suspected and radiographs were taken. Owing to malalignment of the vertebra in both projections at the level of C6to C7, the dog was referred for further investigation.

Vital parameters as well as the results of a haematology and serum biochemical analysis were unremarkable.

The dog was bright and alert without cranial nerve deficits. It showed a weakly ambulatory tetraparesis that was worse on the left side with generalized spinal ataxia. Proprioceptive testing responses were delayed on all four limbs. The segmental spinal reflexes were normal in the pelvic limbs. In both fore limbs, the withdrawal reflex was markedly reduced as well as all the myotatic reflexes (extensor carpi, biceps and triceps). Cutaneous trunci reflex was normal bilaterally. The dog showed a torticollis to the left and a stiff neck. Due to the suspicion of instability and evident spontaneous pain, manipulation and palpation of the neck were not performed. A spinal cord lesion at the level of the C6 to second thoracic (Th2) segments was suspected.

\section{Diagnostic Imaging}

Radiographs taken by the local veterinarian showed a mild ventrodorsal and marked left lateralized rotational subluxation of the vertebral column at the level of C6 to C7. There were no visible fracture lines or bone fragments. The dog was premedicated with methadone (Methadon Streuli, Streuli Pharma AG, Uznach, Schweiz) and midazolam (Dormicum, Roche Pharma AG, Reinach BL, Schweiz). Anaesthesia was induced with propofol (PROPOFOL 1\% MCT Fresenius, Fresenius Kabi AG, Oberdorf, Schweiz) and after intubation maintained with isoflurane, (Isofluran Baxter ad us. vet., Baxter AG, Volketswil, Schweiz) 50\% oxygen and 50\% air. Intravenous fluids (RINGER-LACTAT Fresenius, Fresenius Kabi AG, Oberdorf, Schweiz) were continuously administered $(10 \mathrm{~mL} / \mathrm{kg} /$ hour $)$ for the duration of anaesthesia. Computed tomography (CT) was performed, using a 16-slice helical CT device (Alexion: Toshiba Medical Systems, Oetwil am See, Schweiz). The patient was carefully positioned in dorsal recumbency using a supporting kennel and helical slices of the cervical and cranial thoracic spine were obtained (120 kVp; $100 \mathrm{~mA}$; slice thickness $2.0 \mathrm{~mm}$ reconstructed to $0.5 \mathrm{~mm}$; pitch factor 0.9379; reconstruction bone [WL 1000; WW 3000] and soft tissue algorithm [WL 80, WW 350]). Subsequently, CT myelography (lumbar puncture at L4-L5 with iopamidol $0.2 \mathrm{~mL} / \mathrm{kg}$ ) (Iopamiro 370, Bracco Suisse SA, Bracco, Schweiz) was performed. The retrieved DICOM images were analysed on a workstation using dedicated imaging software (Osirix, Pixmeo SARL, Bernex, Switzerland).

A complete luxation of the left articular process of the $\mathrm{C} 6$ to C7 joint, with the caudal articular process of C6 located ventrally to the cranial articular process of $\mathrm{C} 7$, was observed. Two small bone fragments were located dorsal to the luxated cranial articular facet joint surface of C7. The right articular process joint space $\mathrm{C} 6$ to $\mathrm{C} 7$ was uneven with the caudal part of C6 being rotated and shifted to the left. Homogenous soft tissue attenuating ( $50 \mathrm{HU}$ ) material was present in the left ventral spinal canal at the level of the caudal part of C6. Computed tomography myelography revealed a circumferentially thinned subarachnoid contrast and a moderate dorsoventral flattening of the spinal cord contour secondary to the vertebral malalignment. The aforementioned extradurally located soft tissue in the spinal canal, which was suspected to be epidural haemorrhage or extruded nucleus material, exerted no obvious additional compressive force (-Fig. 1).

\section{Surgery and Postoperative Care}

A skin incision in the dorsal midline from C4 to Th2 was made and a dorsal surgical approach to the cervical vertebral column was performed. ${ }^{8}$ The spinous processes of $\mathrm{C} 6$ and $\mathrm{C} 7$ as well as the articular facets were exposed and the luxated left facet joint with the cartilaginous articular surface of the dorsally displaced cranial articular process of C7 could be seen. Two bone forceps were placed dorsal directly over the lamina into the spinous processes of C6 and C7 and linear traction was applied. With the Hohmann lever placed in between the displaced articular processes, the luxated joint surfaces were distracted and reduced. Due to slight instability of the two articular processes, a tension suture with 6-metric, braided, polyethylene suture (Ethibond 6* Excel, Johnson \& Johnson AG (Johnson \& Johnson Medical), Zug, Schweiz) was placed dorsally between C6 and C7. A $1.5 \mathrm{~mm}$ hole at the base and centre of the spinous process of C6 was drilled and the suture was placed through this hole and caudally around the prominent spinous process of C7. With the presurgically applied subarachnoid contrast still being partially present, a postoperative CT scan was performed. The luxated left caudal articular process of C6 was in situ with residual minimal subluxation. The soft tissue material in the left ventral aspect of the spinal canal was seen caudal to the intervertebral disc (IVD) space C6 to $\mathrm{C7}$, exerting mild compressive force on the spinal cord (-Fig. 2). The dog recovered uneventfully from anaesthesia. Intravenous fluids were continued ( $4 \mathrm{~mL} / \mathrm{kg} /$ hour $)$ for 12 hours. Buprenorphine (Bupaq ad us. vet., Streuli Pharma AG, Uznach, Schweiz) was administered until the fentanyl patch (Durogesic Matrix TTS, Janssen-Cilag AG, Zug, Schweiz) started to take effect. Carprofen (Rimadyl, ad us. vet. Kautabletten, Zoetis Schweiz GmbH, Zürich, Schweiz) was administered the day after and continued for 12 days. The day after surgery, the dog had ambulatory tetraparesis and a stiff neck with reluctance to extend it dorsally in a normal position.

Over the next 10 days, the dog improved to a neurologically normal state. The re-examination at the referring veterinarian with removal of the skin sutures showed no abnormal clinical signs. Follow-up examination 9 months after surgery showed no neurological abnormalities and there was unrestricted motion of the neck.

A plain control CT study was conducted with comparable patient positioning and machine settings and same anaesthesia protocol as used previously. It revealed sustained correct vertebral cervical alignment (-Fig.3), moderate osteoarthritis 


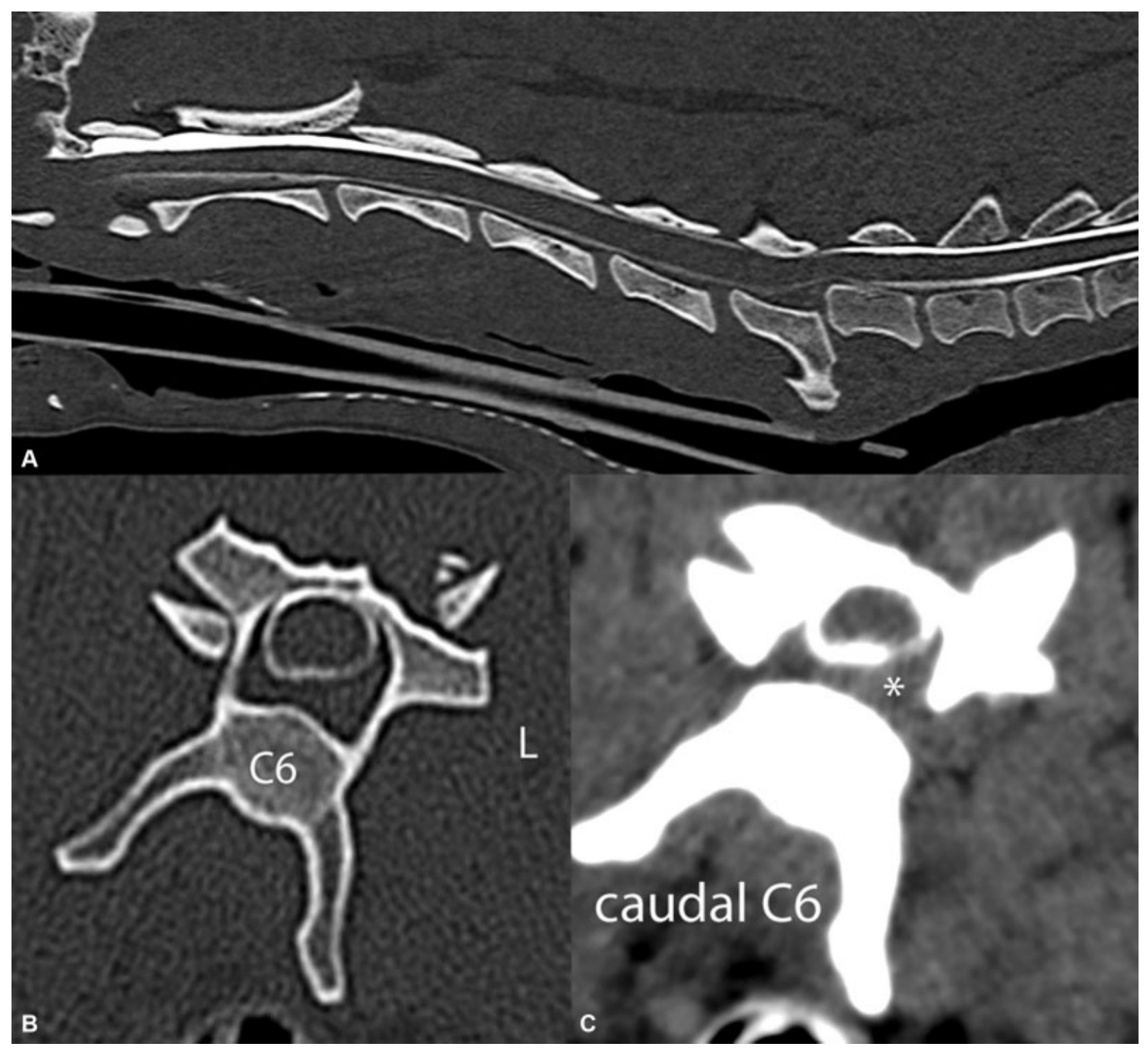

Fig. 1 Computed tomography-myelography of the cervical spine in sagittal view (A) and transverse view at the level of C6 in bone algorithm (B; WL 1000 $\mathrm{HU}, \mathrm{WW} 3500$ ) and soft tissue algorithm (C; WL $90 \mathrm{HU}, \mathrm{WW} 300 \mathrm{HU}$ ). Axial and rotational malalignment of the cervical spine secondary to the left cranial articular process of $\mathrm{C} 7$ being wedged dorsal to the caudal articular process of $C 6$. Soft tissue attenuating material $\left(^{*}\right)$ in the left ventral aspect of the vertebral canal aggravates the displacement and deformation of the spinal cord.

of the right facet joint and mild spondylosis deformans C6 to C7. Continuous rough and solid new bone formation was noted on the dorsal lamina and spinous process of C6 and to a lesser extent of C7. The drill hole in the spinous process of C6 was mildly remodelled, but still readily visible (-Fig. 4). The IVD space C6 to C7 was notably narrowed and the previously described soft tissue material was in a similar manner present in the ventral and left vertebral canal at the same level. It failed to distort the spinal cord or meningeal contour with epidural fat attenuation being maintained circumferentially. However, fat attenuation in the left intervertebral foramen was lost.

\section{Discussion}

Unilateral or bilateral, complete luxation of the facet joint without fracture of the related structures is very rare and has once been mentioned in a review in veterinary medicine. ${ }^{7}$ In our human counterpart, cervical unilateral facet joint dislocation is a well-known phenomenon and accounts for $\sim 6 \%$ of cervical spine injuries in adults, ${ }^{9}$ where the lower cervical spine is more commonly affected in older children and adults. ${ }^{10,11}$

The caudal cervical spine is the most movable part of the vertebral column in dogs. ${ }^{12}$ Mobility of the canine cervical vertebral column increases caudally and C6 to $\mathrm{C} 7$ has been described as the most flexible segment. ${ }^{13}$ The cervical articular processes of the vertebral column are oriented in a horizontal direction to permit primarily lateral movement patterns. In this case, the presumed high-velocity trauma with abrupt stopping and therefore associated flexion, bending and rotation could explain the luxation of the vertebral facet joint. Maximal flexion and simultaneous right-sided bending and rotation lead to distraction and can luxate or 

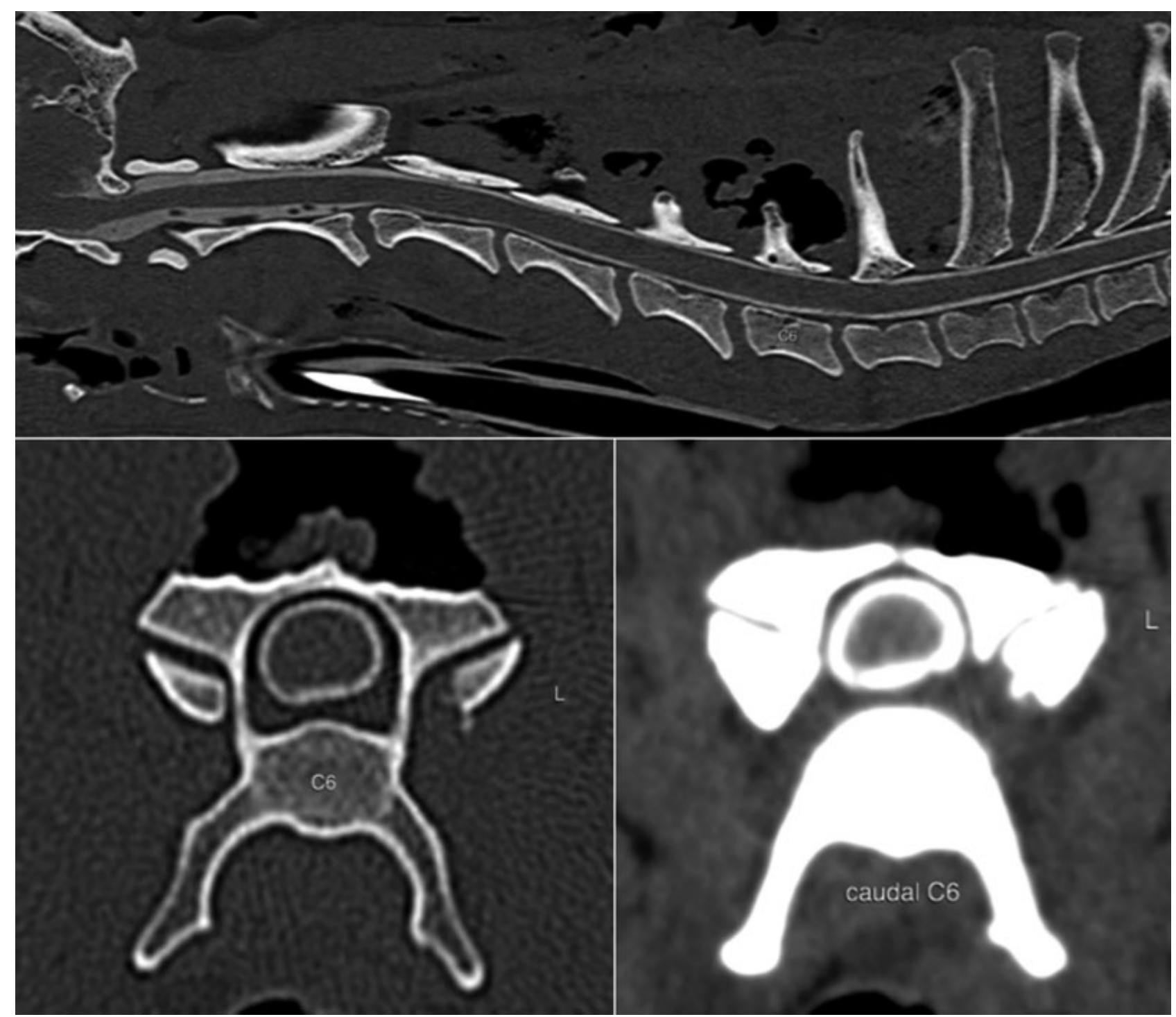

Fig. 2 Post-surgery computed tomography of the cervical spine and residual amount of subarachnoid contrast present. Sagittal and transverse views in comparable setting and location to - Fig. 1 demonstrate achieved spinal re-alignment with residual mild left facet joint subluxation and subtle left ventral extradural soft tissue. The intervertebral disc space C6/7 remains narrow.

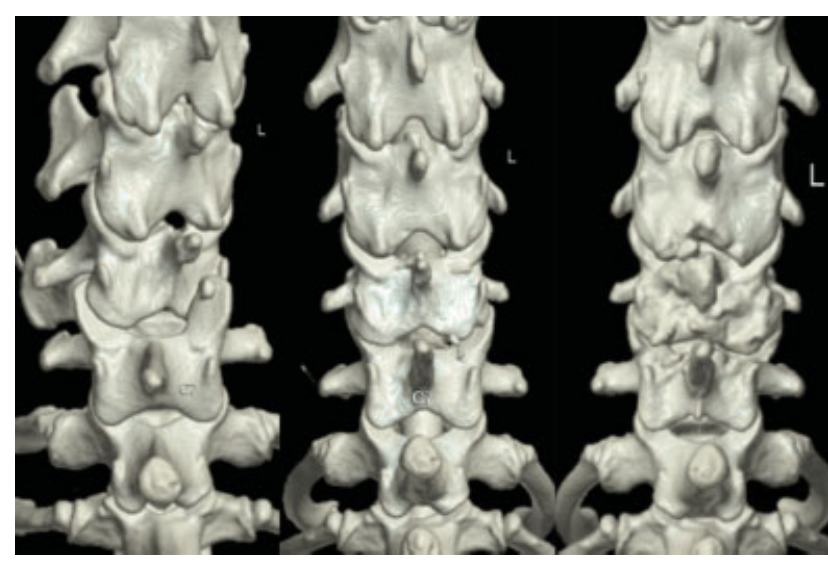

Fig. 3 Three-dimensional reformatted images in dorsal view of the caudal cervical spine; pre-surgically (left), immediate post-surgically (middle) and 9 months post-injury (right). displace the left cranial articular process dorsally to the caudal vertebra of the corresponding cranial one. Due to limited bone stability, such trauma normally results in fracture of the involved vertebral structures and subsequently instability of the affected area.

In our case, overall vertebral morphology of the involved segments was maintained with minimal fragmentation of the left cranial articular process of $\mathrm{C7}$. The caudal articular surfaces of the cervical vertebrae are shorter and generally the facet joints are more oval in shape in large breed dogs than in Dachshunds and small breed dogs. ${ }^{13}$ These may have been predisposing factors in this large breed dog. Malformation or underdeveloped articular processes, that could lead to predisposition for instability and concomitant luxation of a facet joints, were not present.

Dorsal subluxation of the cranial articular process of $C 7$ and its corresponding vertebral body led to marked spinal cord 


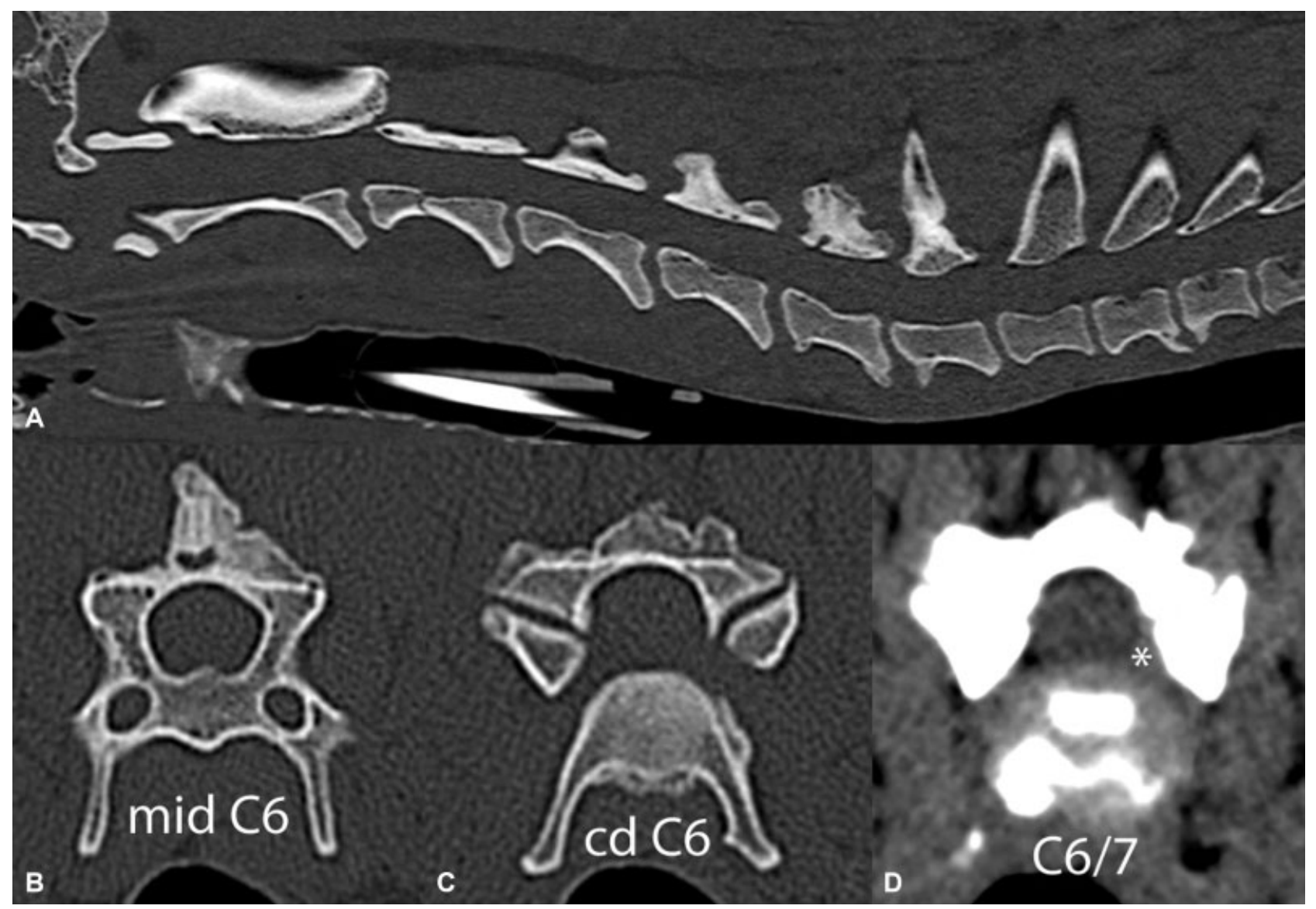

Fig. 4 Plain computed tomography study of the cervical spine in sagittal (A) and transverse (B-D) views. (A) Unchanged reduced dimension of the intervertebral disc space C6/7 with developing spondylosis deformans as well as (D) stationary soft tissue attenuating material $\left({ }^{*}\right)$ in the left ventral spinal canal displacing the local epidural fat and exerting minimal effect on the spinal cord. New bone formation centred on the dorsal lamina and the dorsal spinous process of C6. Visible suture hole at the base of the dorsal spinous process (B).

compression and associated myelopathy. Additionally, an extradurally located soft tissue component at the level of the C7 vertebral body located in ventrolateral position was identified. These findings and the grade of tetraparesis led to the recommendation of surgical reposition to optimize the chance of recovery and to minimize possible sequela. After open reduction in the facet joint, there was still excessive mobility at this side mainly in the sagittal plane due to soft tissue disruption, especially of the facet joint capsule. This led to the decision to place a tension suture dorsally between the spinous processes of $\mathrm{C} 6$ and $\mathrm{C} 7$. Owing to the small spinous process of C6, a burr hole had to be created to guarantee stable anchoring of the suture. This technique aimed to achieve temporary intervertebral reinforcement until periarticular fibrosis would result in permanent stability and reduce the risk of reluxation. The same principle of temporary dorsal stabilization with the goal of long-term stabilization by fibrosis was reported for atlantoaxial subluxation in small breed dogs in a recent study. ${ }^{14}$ The dorsal approach was a practical way to allow inspection and reduction of the affected vertebrae. Marked distraction of the two vertebrae with additional rotation was needed and this would have been challenging with another approach. In contrast to our case management, closed reduction technique has been reported in human pediatrics. ${ }^{15}$ In our case, the chance of a successful closed reduction was consid- ered highly unlikely and potentially harmful. Therefore, we opted for open reduction technique directly and additional sutural stabilization. Application of a neck bandage or splint could therefore be avoided.

The ventrolateral compression of the spinal cord at the level of C7 was not addressed because as it was only mild and the major compression could be relieved with the vertebral reposition. A ventral or lateral approach would have been better for secondary stabilization after reduction and for additional removal of the ventrolateral located material. Due to lack of additional fractures of weight bearing and stabilizing structures, opening of the spinal canal to remove the material in the ventrolateral part was not performed to avoid destabilization of the vertebral column. This material was thought to consist of trauma-related haemorrhage or IVD tissue. The markedly reduced size of the IVD space in the post-surgical CT study leads to the assumption of extruded IVD material. In case of a more pronounced compression, a hemilaminectomy of the luxated facet joint with subsequent decompression would have been chosen.

The CT study 9 months after surgery showed good alignment of the vertebral column, almost normal congruency of the facet joint and only mild osteoarthritis of the right facet joint with no clinical signs of neck pain. The new bone formation on the dorsal lamina and the spinous process of 
C6 and to a lesser extent at C7 was thought to be caused by the polyethylene suture used for temporary stabilization. Due to the aim of temporary stabilization, to reduce the risk of suture-related infection and to avoid this marked bony reaction, an absorbable suture material, for example, polydioxanone of the same size, could have been used. The uneventful recovery and good clinical outcome suggest that the polyethylene suture material used was adequate.

Nine months after surgery, there were no clinical or neurological abnormalities. The CT study showed only minimal degenerative changes secondary to the trauma. The good alignment of the facet joint and the only minimal presence of osteoarthrosis support the good clinical outcome and lower the risk of possible late sequela. Late complications as facet joint arthrosis and neck pain can still not be excluded.

\section{Conflict of Interest}

None declared.

\section{Author Contribution}

Philemon Karli and Danielle Bass contributed to conception of study, study design, acquisition of data and data analysis and interpretation. Martin Bass and Roman Inauen contributed to conception of study, acquisition of data and data analysis and interpretation. All authors drafted, revised and approved the submitted manuscript.

\section{References}

1 Fluehmann G, Doherr MG, Jaggy A. Canine neurological diseases in a referral hospital population between 1989 and 2000 in Switzerland. J Small Anim Pract 2006;47(10):582-587

2 Bali MS, Lang J, Jaggy A, Spreng D, Doherr MG, Forterre F. Comparative study of vertebral fractures and luxations in dogs and cats. Vet Comp Orthop Traumatol 2009;22(01):47-53
3 McKee WM. Spinal trauma in dogs and cats: a review of 51 cases. Vet Rec 1990;126(12):285-289

4 Feeney DA, Oliver JE. Blunt spinal trauma in the dog and cat: insights into radiographic lesions. J Am Vet Med Assoc 1980;16:885-890

5 Selcer RR, Bubb WJ, Walker TL. Management of vertebral column fractures in dogs and cats: 211 cases (1977-1985). J Am Vet Med Assoc 1991;198(11):1965-1968

6 Hawthorne JC, Blevins WE, Wallace LJ, Glickman N, Waters DJ. Cervical vertebral fractures in 56 dogs: a retrospective study. J Am Anim Hosp Assoc 1999;35(02):135-146

7 Jeffery ND. Vertebral fracture and luxation in small animals. Vet Clin North Am Small Anim Pract 2010;40(05):809-828

8 Piermattei DL, Johnson KA. Approach to the caudal cervical and cranial thoracic vertebrae through a dorsal incision. In: Piermattei DL, Johnson KA, eds. An Atlas of Surgical Approaches to the Bones and Joints of the Dog and Cat. 4th edition. Philadelphia: Elsevier; 2004:72-77

9 Lowery DW, Wald MM, Browne BJ, Tigges S, Hoffman JR, Mower WR; NEXUS Group. Epidemiology of cervical spine injury victims. Ann Emerg Med 2001;38(01):12-16

10 Brown RL, Brunn MA, Garcia VF. Cervical spine injuries in children: a review of 103 patients treated consecutively at a level 1 pediatric trauma center. J Pediatr Surg 2001;36(08):1107-1114

11 Kokoska ER, Keller MS, Rallo MC, Weber TR. Characteristics of pediatric cervical spine injuries. J Pediatr Surg 2001;36(01): 100-105

12 Lang J, Loeffler K. Die Bewegungsmoeglichkeiten der Wirbelsaeule von Hund und Katze (The movement abilities of the vertebral column in dogs and cats). Kleintierpraxis 1972;17:217-244

13 Breit S, Künzel W. A morphometric investigation on breedspecific features affecting sagittal rotational and lateral bending mobility in the canine cervical spine (c3-c7). Anat Histol Embryol 2004;33(04):244-250

14 Sánchez-Masian D, Luján-Feliu-Pascual A, Font C, Mascort J. Dorsal stabilization of atlantoaxial subluxation using nonabsorbable sutures in toy breed dogs. Vet Comp Orthop Traumatol 2014;27(01):62-67

15 Chen Y, Wang X, Chen D, Liu X. Surgical treatment for unilateral cervical facet dislocation in a young child aged 22 months old: a case report and review of the literature. Eur Spine J 2013;22 (Suppl 3):S439-S442 